\title{
Intravenous abatacept in Japanese patients with polyarticular-course juvenile idiopathic arthritis: results from a phase III open-label study
}

Ryoki Hara ${ }^{1 *}$, Hiroaki Umebayashi ${ }^{2}$, Syuji Takei ${ }^{3}$, Nami Okamoto ${ }^{4}$, Naomi Iwata ${ }^{5}$, Yuichi Yamasaki ${ }^{3}$, Yasuo Nakagishi ${ }^{6}$, Toshitaka Kizawa $^{7}$, Ichiro Kobayashi ${ }^{8}$, Tomoyuki Imagawa ${ }^{9}$, Noriko Kinjo ${ }^{10}$, Norihito Amano ${ }^{11}$, Yoko Takahashi ${ }^{11}$, Masaaki Mori ${ }^{12}$, Yasuhiko Itoh ${ }^{13}$ and Shumpei Yokota ${ }^{14}$

\begin{abstract}
Background: To investigate efficacy and safety of intravenous abatacept in Japanese patients with active polyarticular-course juvenile idiopathic arthritis ( $\mathrm{pJIA)}$.

Methods: In this phase III, open-label, multicenter, single-arm study, patients with pJIA aged 4-17 years who failed $\geq 1$ biologic or methotrexate received weight-tiered (<75 kg: 10 mg/kg; $75-100$ kg: 750 mg; > 100 kg: 1000 mg) intravenous abatacept at Weeks $0,2,4$, and every 4 weeks thereafter. The study comprised a short-term period (16 weeks) and ongoing long-term period. Primary endpoint: Week $16 \mathrm{JIA-American} \mathrm{College} \mathrm{of} \mathrm{Rheumatology} \mathrm{criteria}$ 30 (JIA-ACR30) response rate. Secondary endpoints/outcomes included Week 16 JIA-ACR50/70/90 response and inactive disease rates, Childhood Health Assessment Questionnaire-Disability Index (CHAQ-DI), pharmacokinetics, safety, and immunogenicity. Proportions of patients achieving Juvenile Arthritis Disease Activity Score in 27 joints using C-reactive protein (JADAS27-CRP) remission (score $<1$ ) and minimal disease activity (MDA; score $<3.8$ ), were among exploratory endpoints.
\end{abstract}

Results: All 20 patients who received study medication completed the short-term period. During the long-term period, two patients discontinued due to insufficient efficacy or patient decision. Median age and disease duration at baseline were 10.5 and 0.75 years, respectively. Week $16 \mathrm{JIA-ACR30} \mathrm{response} \mathrm{rate} \mathrm{(primary} \mathrm{endpoint)} \mathrm{was} \mathrm{90.0 \%}$ (18/20). JIA-ACR50/70/90 response and inactive disease rates at Week 16 were 75.0\% (15/20), 70.0\% (14/20), 35.0\% (7/20), and 25.0\% (5/20), respectively. At Week 52, JIA-ACR30/50/70/90 response and inactive disease rates were observed by $88.9 \%(16 / 18), 88.9 \%(16 / 18), 83.3 \%(15 / 18), 66.7 \%(12 / 18)$ and $44.4 \%(8 / 18)$, respectively. CHAQ-DI improved after Week 12. JADAS27-CRP remission and MDA were achieved by $15.0 \%(3 / 20)$ and $45.0 \%(9 / 20)$ of patients at Week 16, and by 50.0\% (9/18) and 78.0\% (14/18) of patients at Week 52, respectively. The mean abatacept pre-dose serum concentration was above the target therapeutic exposure $(10 \mu \mathrm{g} / \mathrm{ml})$ from Week 8 through Week 16. All adverse events were of mild/moderate intensity, except for one case of severe gastroenteritis. No deaths, malignancies, or autoimmune disorders were observed. No antidrug antibodies were detected through Week 16; one patient had a positive immunogenic response during the cumulative period.

Conclusion: Intravenous abatacept was efficacious and well tolerated in Japanese patients with active pJIA. (Continued on next page)

\footnotetext{
* Correspondence: r.hara.ycuped@gmail.com

${ }^{1}$ Department of Pediatrics, Yokohama City University School of Medicine, 3-9

Fukuura, Kanazawa-ku, Yokohama-shi, Kanagawa 236-0004, Japan

Full list of author information is available at the end of the article
}

(c) The Author(s). 2019 Open Access This article is distributed under the terms of the Creative Commons Attribution 4.0 International License (http://creativecommons.org/licenses/by/4.0/), which permits unrestricted use, distribution, and reproduction in any medium, provided you give appropriate credit to the original author(s) and the source, provide a link to the Creative Commons license, and indicate if changes were made. The Creative Commons Public Domain Dedication waiver (http://creativecommons.org/publicdomain/zero/1.0/) applies to the data made available in this article, unless otherwise stated. 
(Continued from previous page)

Trial registration: ClinicalTrials.gov: NCT01835470. Date of registration: April 19, 2013.

Keywords: Abatacept, Disease-modifying anti-rheumatic drugs (DMARDs), Japanese, Juvenile idiopathic arthritis, Pharmacokinetics

\section{Background}

Juvenile idiopathic arthritis (JIA) is a pediatric rheumatic disease of unknown etiology that presents in children before the age of 16 years [1]. In developed countries, JIA occurs with a prevalence of $16-150$ cases per 100,000 children [1], with the estimated prevalence in Japan being 10-15 cases per 100,000 children [2]. Polyarticular-course JIA (pJIA) is the most common JIA subtype [1,2], and is defined as the presence of $\geq 5$ affected joints within the first 6 months of onset of disease symptoms $[3,4]$.

In Japan, methotrexate (MTX) is the recommended first-line disease-modifying antirheumatic drug (DMARD) therapy for pJIA [2]. If disease activity remains moderate or high following 3 months of MTX treatment, second-line treatment should be initiated with biologic DMARDs, such as a tumor necrosis factor- $\alpha$ inhibitor (TNFi) or interleukin (IL)- 6 receptor blocker $[5,6]$. Although the TNFis etanercept and adalimumab and the IL- 6 receptor blocker tocilizumab have been approved for the treatment of JIA in Japan [7-9], alternative treatment options still need to be investigated for patients who are intolerant or do not respond to available conventional synthetic and biologic DMARDs, or who lose response over time [10-18].

Abatacept is a recombinant fusion protein comprising the extracellular domain of human cytotoxic T-lymphocyte-associated protein-4 (CTLA4) linked to a modified human immunoglobulin (Ig) G1 Fc portion that selectively modulates the $\mathrm{CD} 80 / \mathrm{CD} 86: \mathrm{CD} 28$ costimulatory signal that is required for full T-cell activation [19]. Compared with other currently available treatments for rheumatoid arthritis (RA), abatacept has a fundamentally different mechanism of action from other biologic DMARDs, as it targets the underlying process of T-cell activation [20,21]. Abatacept has been shown to reduce disease progression, and improve function and health-related quality of life in RA [22-24]. The intravenous (IV) formulation of abatacept is effective and well tolerated in PJIA $[12,15,25,26]$, and has been approved for the treatment of active pJIA in patients over 2 years old in the USA [20] and over 6 years old in Canada [27] and Europe [28]. In Japan, IV abatacept is approved for the treatment of adult patients with RA and in February 2018, approval was granted for the treatment of pediatric patients with pJIA [29].

This study was designed to investigate the efficacy, pharmacokinetics (PK), safety, and immunogenicity of
IV abatacept in Japanese patients with pJIA. Here, the 1-year interim results are presented. To our knowledge, this is the first publication of data for abatacept treatment in Japanese patients with pJIA.

\section{Patients and methods \\ Study design}

This single-arm, open-label, multicenter, two-part, phase III study (NCT01835470) was initiated in September 2013 and conducted across 13 centers in Japan. During the 16-week short-term period, patients received IV abatacept at Week 0 (Day 1), Week 2 (Day 15), Week 4 (Day 29), and then every 4 weeks (Q4W) at a dose based on the patient's weight at each visit (<75 kg: $10 \mathrm{mg} / \mathrm{kg} ; 75-100 \mathrm{~kg}$ : $750 \mathrm{mg}$; > $100 \mathrm{~kg}: 1000 \mathrm{mg}$ ), with or without MTX (4-10 $\mathrm{mg} / \mathrm{m}^{2} /$ week). After completion of the short-term period, patients entered the long-term period, during which they continued to receive IV abatacept Q4W until approval of abatacept for the treatment of pJIA in Japan, or termination of abatacept development by the study sponsor (Bristol-Myers Squibb K.K.). Here we report efficacy and PK data for the short-term period (up to Week 16 [Day 113]) and cumulative efficacy and safety data for the shortand long-term periods combined; cumulative efficacy data are reported to study Week 52 (Day 365), and cumulative safety data are reported to study Week 136 (Day 953). The long-term period is ongoing for most patients.

During the short-term period, the use of MTX (4-10 $\mathrm{mg} / \mathrm{m}^{2} /$ week) or low-dose oral corticosteroids $(\leq 10 \mathrm{mg} /$ day or $\leq 0.2 \mathrm{mg} / \mathrm{kg} /$ day, whichever was less) and non-steroidal anti-inflammatory drugs was permitted at stable doses, although the dose of these agents could be reduced if an adverse event (AE) occurred and subsequently increased to the original starting dose if that $\mathrm{AE}$ resolved. During the cumulative period, the use of all conventional synthetic and biologic DMARDs was prohibited, with the exception of MTX. Patients who completed the long-term period or who discontinued abatacept treatment early during the short-term or long-term periods subsequently entered a follow-up period and were assessed at 4, 12, and 24 weeks after their last dose of study medication. Patients who started commercial abatacept were not permitted to enter the follow-up period.

\section{Patients}

Eligible patients were Japanese, aged 4-17 years, and met the International League of Associations for Rheumatology 
criteria for JIA [3], with a diagnosis of one of the following: extended oligoarticular JIA, polyarthritis rheumatoid factor (RF) positive, polyarthritis RF negative, or systemic JIA with a polyarticular course and absence of systemic features within the 6 months prior to enrollment. Patients were required to have a history of $\geq 5$ active joints with active disease and active articular disease at baseline, defined as $\geq 2$ active joints and $\geq 2$ joints with limitation of motion (LOM), and inadequate therapeutic response or intolerance to $\geq 1$ biologic DMARD or MTX, as determined by the examining physician.

Patients were excluded if they had systemic onset JIA with any of the following manifestations within the 6 months prior to enrollment: intermittent fever due to JIA, rheumatoid rash, hepatosplenomegaly, pleuritis, pericarditis, or macrophage activation syndrome. $\mathrm{Pa}-$ tients with another rheumatic disease or major chronic infectious, inflammatory or immunologic disease (e.g. psoriatic arthritis, inflammatory bowel disease, spondyloarthropathy, hypogammaglobulinemia, or systemic lupus erythematosus) were also excluded from the study.

\section{Study endpoints}

The primary study endpoint was JIA-American College of Rheumatology (ACR) criteria 30\% improvement (JIA-ACR30) response rate at Week 16. JIA-ACR30 was defined as $a \geq 30 \%$ improvement in at least three of the six JIA-ACR core set variables and a $>30 \%$ worsening in no more than one of the six JIA-ACR core set variables [30]. Inactive disease (modified criteria) was defined as no active joints, a Physician's Global Assessment (PGA) score of $\leq 10 \mathrm{~mm}$, and a C-reactive protein (CRP) value of $\leq 0.3 \mathrm{mg} / \mathrm{dL}$ [31]. Secondary study endpoints and outcomes were JIA-ACR50, 70, and 90 response rates [14], inactive disease rate at Week 16, and physical function measured using the Disability Index of the Childhood Health Assessment Questionnaire (CHAQ-DI) [32] at Week 16, as well as PK, safety and tolerability, and immunogenicity during the short-term period. Exploratory endpoints and outcomes included JIA-ACR30, 50, 70, and 90 response rates, inactive disease rate, juvenile arthritis disease activity score 27 active joint count-CRP (JADAS27-CRP) remission rate [33-36], long-term safety during the cumulative period, and immunogenicity during the cumulative period and 6 months following discontinuation of treatment. The Institute of Clinical Outcomes Research and Education (ICORE, Woodside, CA, USA) provided the licensing to use the CHAQ-DI in this study [32].

\section{Efficacy assessment}

Efficacy assessments were performed before administration of study medication at each visit. The following six JIA-ACR core set variables were assessed [37]: number of active joints, number of joints with LOM, PGA of disease activity (scale: $0-100 \mathrm{~mm}$ ), Parental Global Assessment of patient overall well-being (PaGA; scale: 0-100 $\mathrm{mm}$ ), physical functional as measured by the CHAQ-DI (scale: 0-3; completed by the patient or their parent/ caregiver), and laboratory measure of inflammation, as measured by serum CRP level.

JADAS27-CRP was calculated as the sum of the scores of the following four components: PGA of disease activity, PaGA of overall well-being, active joint count in 27 joints, and CRP level. Remission was defined as JADAS27-CRP $<1$, and minimal disease activity was defined as JADAS27-CRP $<3.8$.

\section{PK assessment}

The serum abatacept concentration was determined using a sensitive and validated enzyme-linked immunosorbent assay (Covance Inc., Trenton, NJ, USA), using a biotinylated monoclonal mouse anti-human CTLA4 antibody (clone 11D4), as described previously [38]. The validated linear assay range was $1.0-30.0 \mathrm{ng} / \mathrm{mL}$. The PK parameters assessed were pre-dose observed serum concentration $\left(\mathrm{C}_{\text {trough }}\right)$ measured at Weeks $2,4,8,12$, and 16 , and maximum observed serum concentration $\left(C_{\max }\right)$ measured at Weeks 8, 12, and 16.

\section{Safety assessment}

Safety was evaluated during the short-term, long-term, and follow-up study periods by monitoring of AEs and laboratory tests. All AEs were coded using the Medical Dictionary for Regulatory Activities version (MedDRA) 19.0. Causality assessment between each AE and study medication was performed by the investigator.

\section{Immunogenicity assessment}

Serum samples were collected prior to administration of the study medication at Weeks 0,8 , and 16 during the short-term period, and Weeks 32 and 52, then every 6 months, during the long-term period. A validated sensitive electrochemiluminescence assay was used to detect and evaluate anti-drug antibodies (ADA) specific to 'CTLA4 \pm Ig' and 'Ig and/or junction region' in serum samples [38, 39]. A sample was considered seropositive if immunodepletion was observed with abatacept or truncated CTLA4 and reported as positive with a titer of $\geq 10$.

\section{Statistical analyses}

There was no formal statistical hypothesis or testing for this study. All patients who received at least one dose of study medication were included in the efficacy, PK, safety, and immunogenicity analyses. For the JIA-ACR30, 50, 70, and 90 response rates, and inactive disease status analyses, two-sided 95\% confidence intervals (CIs) were computed using an exact method based on the binomial distribution. 
For the short-term period efficacy analysis, any patient who prematurely discontinued the trial after receiving study medication had missing data imputed as an ACR non-response at all scheduled protocol visits up to Week 16 subsequent to the point of discontinuation. The cumulative period efficacy analysis was performed based on as-observed data. The proportion of JIA-ACR30 responders was summarized at Week 16 for the following subgroups: gender, baseline age, JIA subtype, concomitant MTX therapy, and prior biologic treatment. PK summary statistics of mean and standard deviation were presented for $\mathrm{C}_{\text {trough }}$ and $\mathrm{C}_{\max }$ by day in the short-term period. AEs, serious AEs (SAEs), deaths, discontinuations due to AEs, clinical laboratory abnormalities, changes in vital signs, and positive immunogenicity rates were summarized.

\section{Sample size}

The sample size of 20 patients was determined based on the operational feasibility of a local JIA study. In a preceding JIA study (NCT00095173), JIA-ACR30 response rate was $63.7 \%$. Based on the assumption of a JIA-ACR30 response rate of $65 \%$, a sample size of 20 treated patients would provide a two-sided exact 95\% CI of 40.8 to $84.6 \%$.

\section{Results}

\section{Patients}

A total of 23 patients were enrolled in the study, three of whom were excluded as they no longer met study criteria (Fig. 1). All 20 patients who received treatment completed the short-term period and subsequently entered the long-term period. At Week 52, 2/20 (10\%) patients had discontinued treatment during the long-term period: one withdrew consent and did not enter the follow-up period, the other discontinued due to lack of efficacy and completed the follow-up period. Most patients were female, with a median baseline age of 10.5 years and disease duration of $<1$ year (Table 1); at baseline, $80 \%$ of patients were receiving concomitant MTX and 20\% had previously taken one or more biologic DMARD(s). Twelve of 20 patients $(60 \%)$ from the overall population received $>24$ infusions of abatacept over the cumulative period (i.e. to Week 52 from last enrolled patient's first treatment).

\section{Efficacy}

The proportion of patients who achieved JIA-ACR30, 50, 70 and 90 response, and inactive disease over time from baseline to Week 52 of the cumulative period are shown in Fig. 2. At Week 16, 18/20 (90\%) patients achieved a JIA-ACR30 response (primary endpoint). JIA-ACR50, 70, and 90 response rates, and inactive disease rate were 75.0, $70.0,35.0$, and $25.0 \%$, respectively. During the cumulative period to Week 52, JIA-ACR30 and 50 response rates increased progressively from Week 2 (first assessment) to Week 16 (end of the short-term period) and remained high to Week 52 (Fig. 2). JIA-ACR70 and 90 response rates and inactive disease rate also gradually increased to Week 16 followed by a sustained improvement to Week 52 (Fig. 2). At Week $52(n=18)$, the JIA-ACR30, 50, 70, and 90 response rates, and inactive disease rate were 88.9, $88.9,83.3,66.7$, and $44.4 \%$, respectively. In the subgroups analyses, no marked apparent differences were observed on JIA-ACR30 response rates at Week 16 regardless of sex or age at baseline, disease subtype at study entry, concomitant MTX at study Day 1 or prior biologic DMARD therapy (data not shown). However, due to the small sample size of 20 patients in this study, these data should be interpreted carefully and with caution.

All six JIA-ACR core set variables improved from baseline to Week 16 and throughout the cumulative period to Week 52 (Fig. 3). Rapid improvement, as early as Week 2, was observed for number of active joints, number of joints with LOM, PGA score, and CRP level. The improvement observed for active joints and joints with LOM plateaued at Week 28 but was sustained

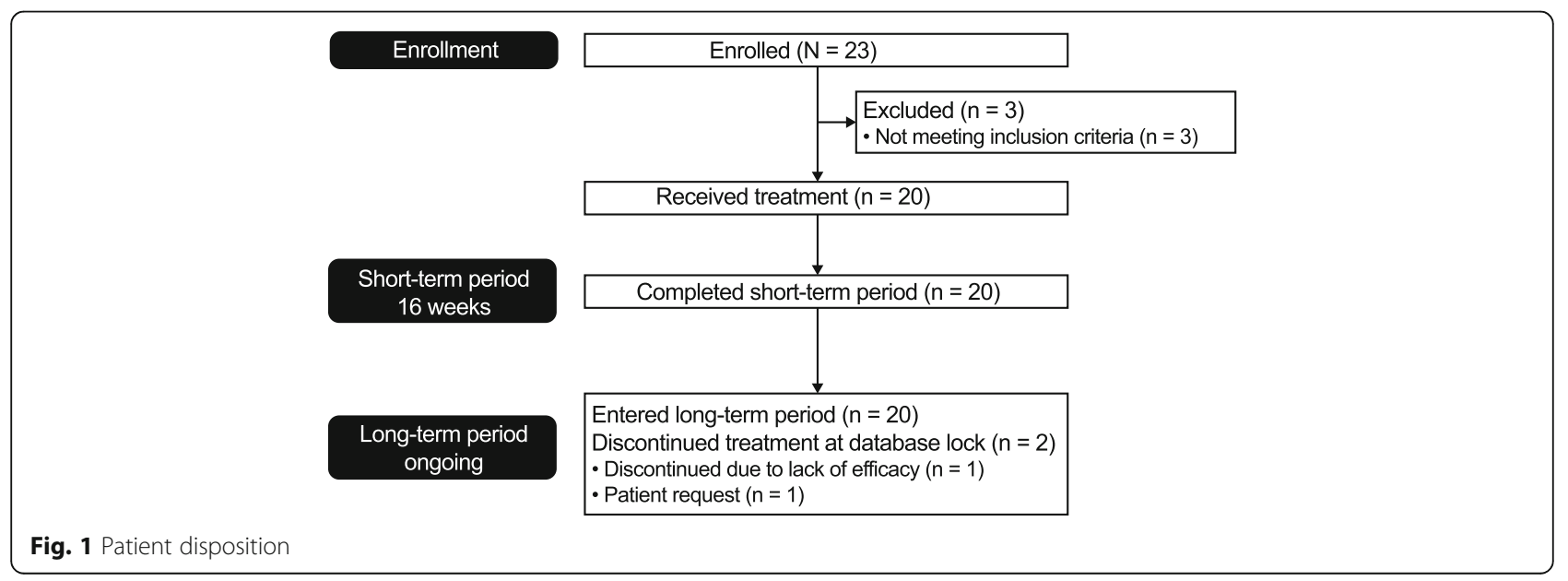


Table 1 Baseline demographics and patient characteristics

\begin{tabular}{|c|c|}
\hline Baseline characteristic & $\begin{array}{l}\text { Patients } \\
(N=20)\end{array}$ \\
\hline Age, years & $10.5(5-16)$ \\
\hline \multicolumn{2}{|l|}{ Age group, n (\%) } \\
\hline $4-8$ years & $8(40)$ \\
\hline $9-12$ years & $7(35)$ \\
\hline $13-17$ years & $5(25)$ \\
\hline Weight, kg & $37.9(15.4-68.3)$ \\
\hline Female, n (\%) & $15(75)$ \\
\hline Race: Japanese, n (\%) & $20(100)$ \\
\hline Disease duration, years & $0.75(0.2-11.9)$ \\
\hline Number of active joints & $6.0(2.0-19.0)$ \\
\hline Number of joints with LOM & $4.0(2.0-10.0)$ \\
\hline Parent Global Assessment, VAS 100 mm & $37.5(0-94.0)$ \\
\hline Physician Global Assessment, VAS 100 mm & $37.0(10-80.0)$ \\
\hline CHAQ-DI & $0.63(0-2.88)$ \\
\hline CRP, mg/dL & $0.58(0.02-2.67)$ \\
\hline JADAS27-CRP & $12.0(4.2-26.4)$ \\
\hline \multicolumn{2}{|l|}{ JIA disease subtype at study entry, $\mathrm{n}(\%)$} \\
\hline Extended oligoarticular & $2(10)$ \\
\hline Polyarticular (RF negative) & $8(40)$ \\
\hline Polyarticular (RF positive) & $10(50)$ \\
\hline Systemic & 0 \\
\hline Prior biologic therapy, n (\%) & $4(20)$ \\
\hline Tocilizumab & $3(15)$ \\
\hline Anti-TNF therapy & $4(20)$ \\
\hline Adalimumab & $4(20)$ \\
\hline Etanercept & $1(5)$ \\
\hline Concomitant MTX therapy at Day 1, n (\%) & $16(80)$ \\
\hline MTX dose, $\mathrm{mg} / \mathrm{m}^{2} /$ week & $8.9(6.0-10.3)$ \\
\hline
\end{tabular}

Data are median (minimum-maximum) unless specified otherwise CHAQ-DI Childhood Health Assessment Questionnaire-Disability Index, $J A D A S 27-C R P$ juvenile arthritis disease activity score 27 active joint count-Creactive protein, JA juvenile idiopathic arthritis, LOM limitation of motion, MTX methotrexate, $R F$ rheumatoid factor, $S D$ standard deviation, $T N F$ tumor necrosis factor, VAS visual analog scale

thereafter out to Week 52, whereas improvements in PGA and CRP continued to increase up to Week 52. Little, if any, improvement in CHAQ-DI or PaGA was observed during the early phase of the study (within 12 weeks of starting treatment with abatacept); however, both parameters started to show continuous improvement after 12 weeks of treatment.

At baseline, mean JADAS27-CRP score was 13.9, with no patients in remission (JADAS27-CRP score $<1$ ) or with minimal disease activity (JADAS27-CRP score < 3.8). Mean JADAS27-CRP score gradually decreased over time from baseline to Week 52 (Fig. 4a), with a mean change from baseline in JADAS27-CRP score of -

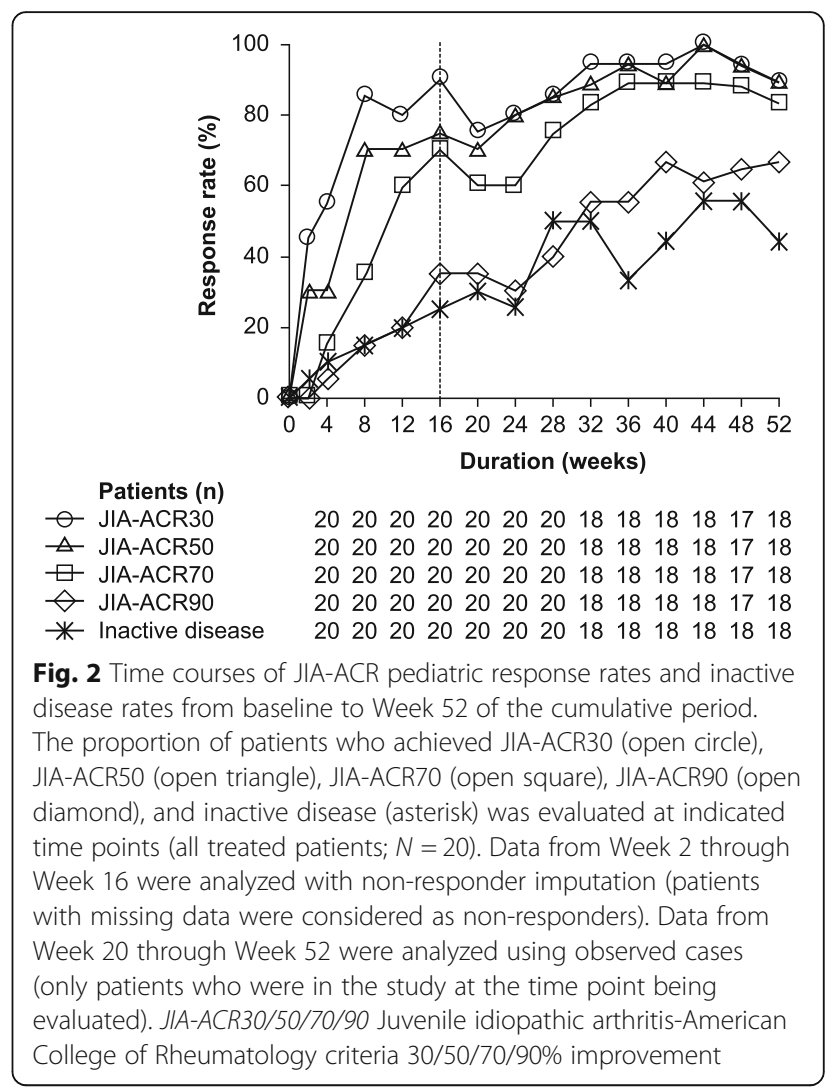

8.7 at Week 16 , and -10.8 at Week 52 . Remission was achieved in 3/20 (15\%) patients at Week 16 and in 9/18 (50\%) patients at Week 52 (Fig. 4b). Minimal disease activity was reported in $9 / 20(45 \%)$ patients at Week 16 and 14/18 (78\%) patients at Week 52 (Fig. 4b).

\section{Pharmacokinetics}

Mean $\mathrm{C}_{\text {trough }}$ serum abatacept levels increased from Week 2 to Week 4, then decreased to Week 8. $C_{\text {trough }}$ levels were maintained at $17-18 \mu \mathrm{g} / \mathrm{mL}$ from Week 8 for the remainder of the short-term period (Fig. 5). Mean $\mathrm{C}_{\max }$ levels were maintained from Week 8 to 16 (Fig. 5).

\section{Safety}

During the short-term period, all patients had at least one $\mathrm{AE}$, all of which were mild or moderate in intensity; treatment-related AEs were observed in 5/20 (25\%) patients (Table 2). Infections were the most commonly reported AEs in the short-term period, of which the most frequent were nasopharyngitis $(n=5[25 \%])$ and pharyngitis $(n=5$ [25\%]). All other AEs across all MedDRA preferred terms were reported in no more than two patients. Two patients experienced SAEs (gastroenteritis [mild, related to study drug] and an exacerbation of a pre-existing disease [mild, unrelated to study drug]). No patients were diagnosed with a malignancy or an autoimmune disorder, other than the aforementioned exacerbation of a pre-existing disease. 
CHAQ-DI

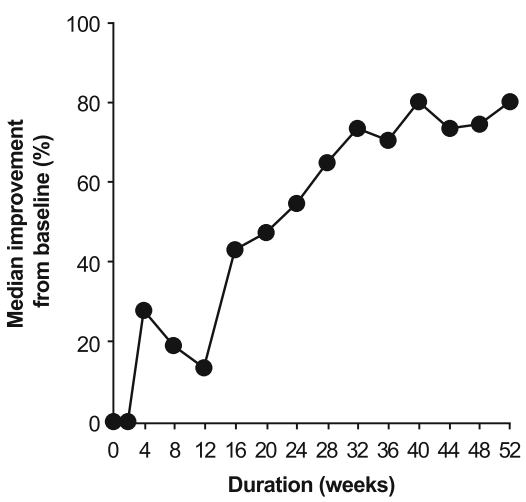

Patients (n) $\quad 20202020202020181818181718$

Joints with LOM

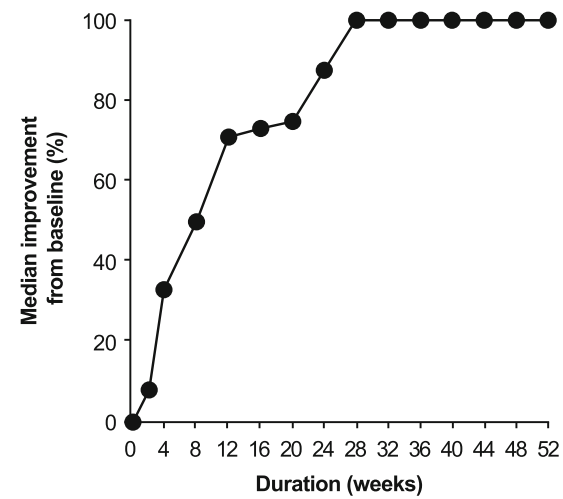

Patients (n) $\quad 2020202020202018 \quad 1818 \quad 1818 \quad 18$

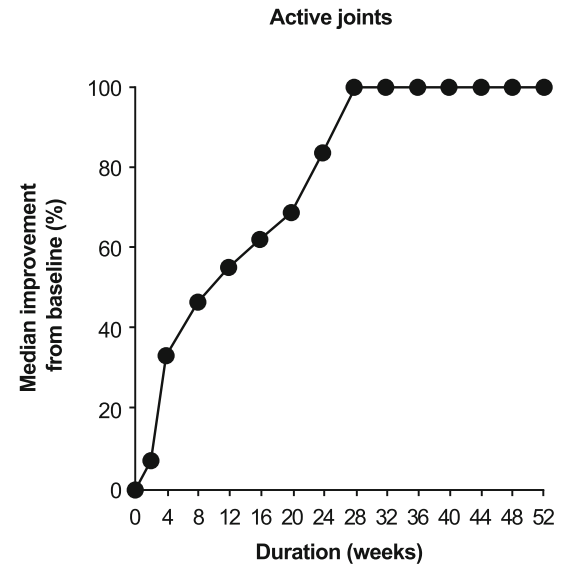

Patients (n) $\quad 20202020202020181818181818$

PGA

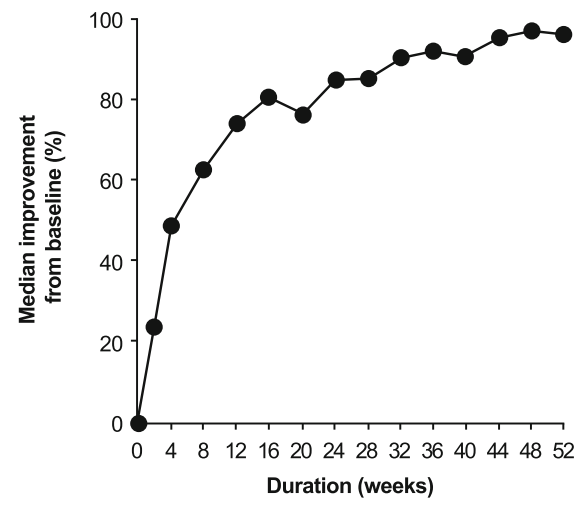

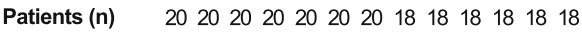

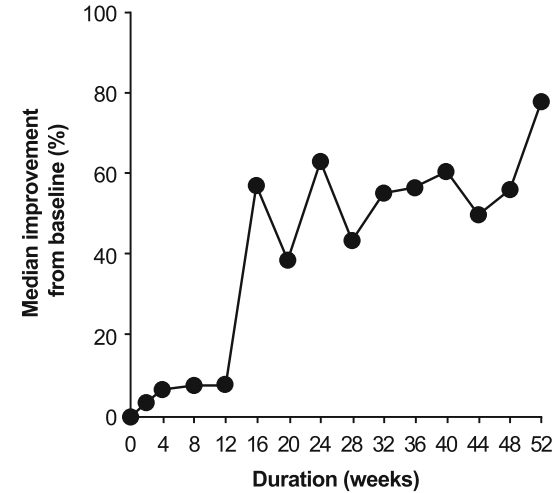

Patients (n) $\quad 202020202020201818181817 \quad 18$

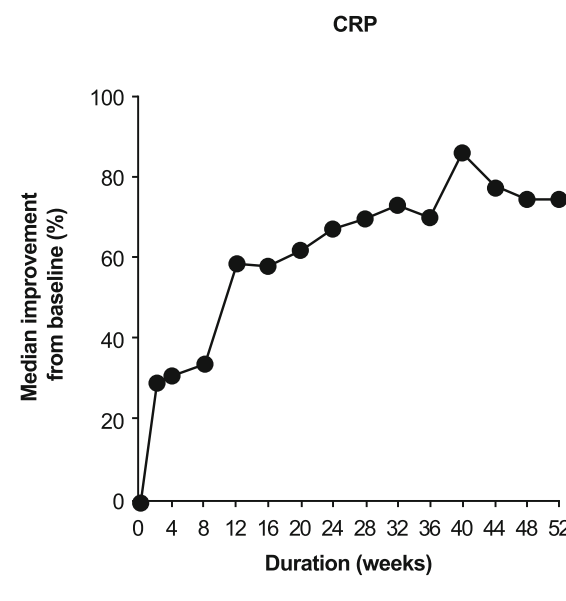

Patients (n) $\quad 2020202020202018 \quad 1818 \quad 18 \quad 18 \quad 18$

Fig. 3 Time course of JIA-ACR core set variables improvement from baseline to Week 52 of the cumulative period. The six JIA-ACR core set variables were evaluated as the median (\%) improvement from baseline at indicated time points (all treated patients; $N=20$ ). CHAQ-DI Childhood Health Assessment Questionnaire-Disability Index, CRP C-reactive protein, JIA-ACR Juvenile idiopathic arthritis-American College of Rheumatology criteria, LOM limitation of motion, PaGA Parental Global Assessment of patient overall well-being, PGA Physician Global Assessment 


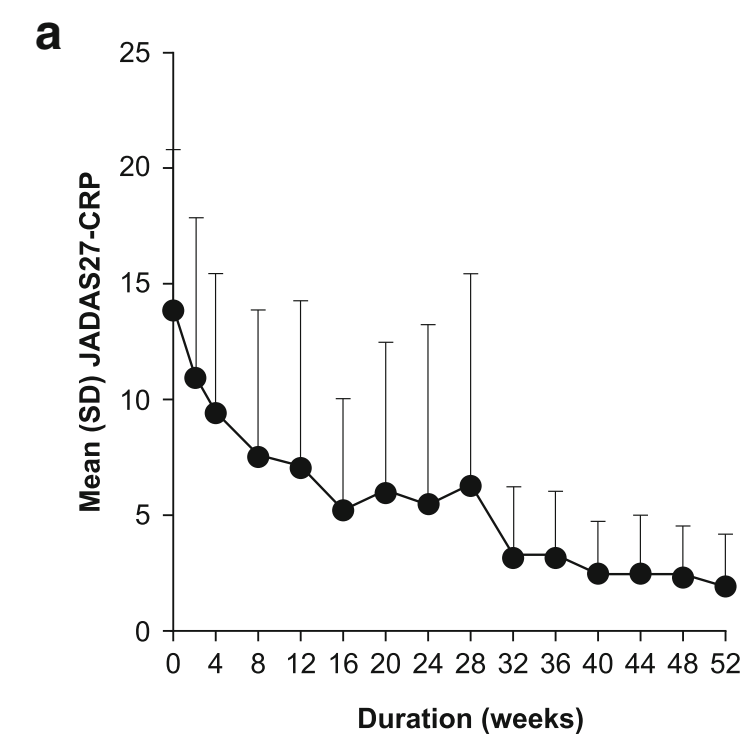

Patients (n) $\quad 20202020202020181818181718$

b

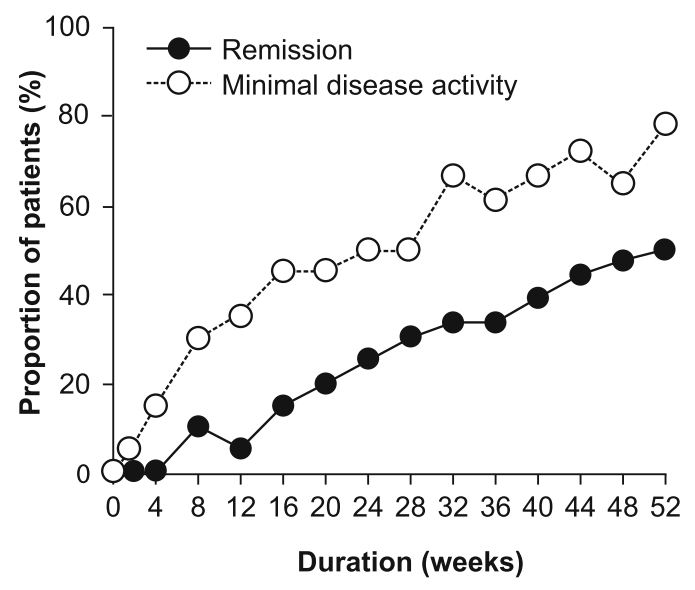

Patients (n) 20202020202020181818181718

Fig. 4 Time course of JADAS27-CRP from baseline to Week 52 of the cumulative period. a Mean (SD) JADAS27-CRP score and (b) the proportion of patients in remission (JADAS27-CRP $<1$, closed circles) or with minimal disease activity (JADAS27-CRP $<3.8$, open circles) from baseline to Week 52 of the cumulative period were evaluated at indicated time points (all treated patients; $N=20$ ). Data were analyzed using observed cases (only patients who were in the study at the time point being evaluated). JADAS27-CRP juvenile arthritis disease activity score 27 active joint count-C-reactive protein, SD standard deviation

There were no discontinuations due to AEs, and no deaths occurred during the short-term period.

During the cumulative period, all AEs were mild or moderate in intensity except for one case of severe gastroenteritis. The most common AEs were infections, of which the most frequent were nasopharyngitis $(n=14)$,

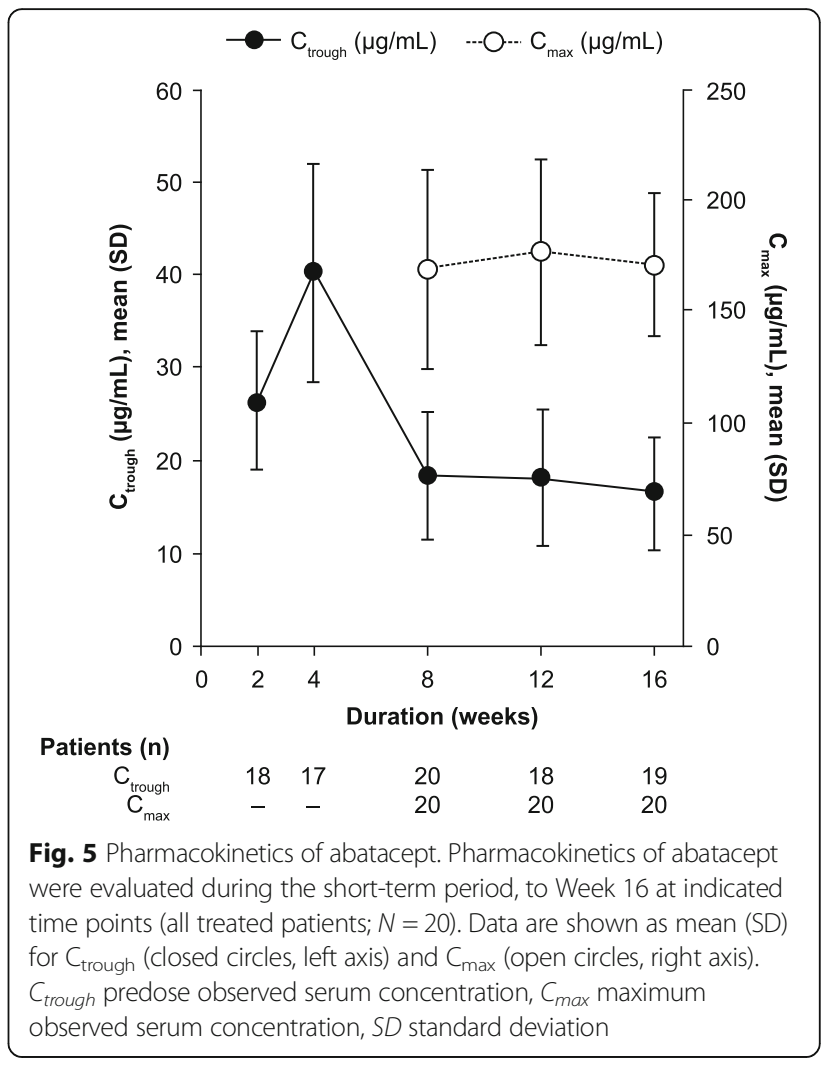

pharyngitis $(n=10)$, and influenza $(n=7)$. SAEs were reported in four patients (gastroenteritis [severe, related to study drug, occurred on Days 21 and 362], varicella [mild, unrelated to study drug, occurred on Day 246], viral tonsillitis [moderate, unrelated to study drug, occurred on Day 575], and an exacerbation of a pre-existing pJIA [mild, unrelated to study drug, occurred on Day 58]). No malignancies or additional autoimmune disorders were reported during the cumulative period. There were no deaths or discontinuations due to AEs (Table 2).

\section{Immunogenicity}

During the short-term period, none of the patients exhibited a positive signal for ADAs. During the cumulative period, one patient tested positive for antibodies to abatacept CTLA4 \pm Ig, at Weeks 32 and 52, with titers of 304 and 476, respectively. This patient had JIA-ACR30, 50, and 70 but not JIA-ACR90 responses in the short-term and long-term periods. No association was observed between positive immunogenicity and loss of efficacy, safety signals, or PK in this patient.

\section{Discussion}

In this phase III study of IV abatacept therapy in Japanese patients with pJIA and inadequate response or intolerance to biologic DMARDs or MTX therapy, the primary endpoint of JIA-ACR30 response at Week 16 
Table 2 Patients with adverse events reported during the short-term period up to Week 16 and cumulative period (all treated patients; $N=20$ )

\begin{tabular}{|c|c|c|}
\hline Safety events & Short-term period (Week 16) & Cumulative period \\
\hline Deaths $^{a}$ & 0 & 0 \\
\hline SAES ${ }^{b}$ & $2(10)$ & $4(20)$ \\
\hline Related SAEs & $1(5)$ & $1(5)$ \\
\hline Discontinued study treatment due to AEs & 0 & 0 \\
\hline AEs & $20(100)$ & $20(100)$ \\
\hline Related AEs & $5(25)$ & $6(30)$ \\
\hline \multicolumn{3}{|l|}{ AEs of special interest } \\
\hline Infections & $16(80)$ & $20(100)$ \\
\hline Malignancies & 0 & 0 \\
\hline Autoimmune disorders ${ }^{c}$ & $1(5.0)$ & 0 \\
\hline \multicolumn{3}{|l|}{ Infusion reactions } \\
\hline Acute infusional & 0 & $1(5)$ \\
\hline Peri-infusional $^{d}$ & $2(10)$ & $5(25)$ \\
\hline Other AEs within $24 \mathrm{~h}^{\mathrm{e}}$ & $4(20)$ & $12(60)$ \\
\hline
\end{tabular}

The short-term period includes data up to 56 days after the last dose in the short-term period or start of the long-term period, whichever occurred first The cumulative period includes data from the first dose in the short-term period up to 56 days post the last dose in the cumulative period ${ }^{a}$ Data include deaths reported during each period including those that occurred $>56$ days after the last dose

bSAEs include hospitalizations for elective surgical procedures

'This event was not new onset, but worsening of the underlying disease (JIA)

${ }^{\mathrm{d}}$ Defined as AEs that occurred during the first 24 hours after the start of abatacept infusion and are included in the pre-specified Medical Dictionary for Regulatory Activities list of peri-infusional events of interest

e Defined as AEs that occurred after the start of abatacept infusion but are not included in the list of peri-infusional AEs of special interest

$A E$ adverse event, JIA juvenile idiopathic arthritis, SAE serious adverse event

was achieved, with $90 \%$ of patients responding. For a sample size of 20 patients, a JIA-ACR30 response rate of $65 \%$ (two-sided exact $95 \%$ CI of 40.8 to $84.6 \%$ ) was predicted based on a previous phase III study of IV abatacept in patients with active pJIA (NCT00095173) [26]. In the current study, IV abatacept had a beneficial effect on physical function, as demonstrated by improvements in CHAQ-DI relative to baseline measurements, and was well tolerated over the 52-week cumulative period.

In addition to meeting the primary efficacy endpoint (JIA-ACR30 response), JIA-ACR50 and 70 responses were also observed as early as Week 2 and response rates continued to increase through to Week 16, plateauing around Week 36. The rates of more stringent JIA-ACR response measures and inactive disease status increased steadily over time with continued abatacept treatment during the cumulative period, similar to the kinetics observed for improvement in JIA-ACR30 response rates. In the international, multicenter, phase III clinical trial and real-world study of IV abatacept, sustained increase in the JIA-ACR response rates during abatacept treatment was also demonstrated. However, in this study, the JIA-ACR30, 50, 70 and 90 response rates, and inactive disease status at week 16 were numerically higher than the corresponding response rates observed in the international trial $(65,50,28,13$ and $13 \%$, respectively) [26]. Importantly, in this study, the patients may have had an early disease onset, with numerically lower disease duration, number of active joints and CHAQ-DI at baseline than those reported in the international study, which may support the notion of a potential effect of patient background, specifically race and ethnicity, on response to abatacept treatment and emphasize the importance of establishing the efficacy and safety profile of the drug in specific patient populations. In addition, the JIA-ACR90 response of $66.7 \%$ at Week 52 observed in this study was similar to that observed in a study of the IL-6 receptor inhibitor tocilizumab in pJIA, in which JIA-ACR90 response was $64.7 \%$ at Week 48 [40].

The present findings are also consistent with those from previous studies that assessed the efficacy of abatacept based on JIA-ACR30 response in different JIA subtypes (extended oligoarticular JIA and RF-positive and RF-negative polyarticular JIA) $[12,15,19,41]$. In subgroup efficacy analyses, there were no marked differences in JIA-ACR30 response rates regardless of sex or age at study baseline, JIA subtype, concomitant MTX dose, or prior biologic therapy. However, it should be noted that statistical difference was not formally analyzed in these subgroups and limited conclusions can be drawn due to the very small sample sizes. JIA-ACR30 response rates achieved with abatacept have previously been found to be unaffected by JIA subtype, but may be lower in patients who have received prior anti-TNF 
therapy compared with those who were anti-TNF naïve at abatacept initiation [26]. The improvement from baseline in each of the six JIA-ACR core set variables during the cumulative period supports the increased overall JIA-ACR response rates. Gradual and continuous improvements were seen in several measures of disease activity, including CHAQ-DI scores, suggesting that abatacept treatment is associated with a reduction in physical disability. These results concur with previous reports of abatacept treatment in patients with pJIA from outside Japan $[15,26]$. Interestingly, in this study, improvement in PaGA was slower and of a lower magnitude than that in PGA over time, which may be a reflection of parents having a worse perception of their child's condition, or higher expectations of a new investigational drug, than the treating physician. These findings, suggesting that parents might overestimate a child's condition, are somewhat consistent with those from a previous study in which children with JIA reported that their health-related quality of life was better than that reported by their parents [42]. It should be noted that PGA, which was evaluated by objective parameters such as joint symptoms and CRP, promptly improved, implying that it might not be appropriate to make a decision for insufficiency of the therapeutic intervention based on only PaGA and CHAQ (although the decision is usually made 3 months after starting the treatment). It should be taken into account that PaGA and PGA measure different aspects of disease and should not be considered redundant.

JADAS has been recognized recently as a valuable measure of disease activity within the clinical trials and routine practice, as it allows for a more accurate determination of the disease activity state than JIA-ACR response criteria [43]. Mean score and change from baseline in JADAS27-CRP, as well as proportion of patients in JADAS27-CRP-defined remission and with minimal disease activity, exploratory endpoints in this trial, were investigated to determine the disease activity state of patients with pJIA over the study period. [43] In this study, proportion of patients with JADAS27-CRP-defined remission (JADAS27-CRP score $<1$ ) and with minimal disease activity (JADAS27-CRP score $<3.8$ ) increased over time with abatacept treatment. Importantly, the mean change from baseline in JADAS27-CRP score decreased early and remained low over time with abatacept therapy, reflecting the rapid and sustained response to abatacept in this patient population.

A PK steady state was achieved by Week 8 , with $\mathrm{C}_{\text {trough }}$ levels maintained above the target therapeutic level of $10 \mu \mathrm{g} / \mathrm{mL}$, which has been associated with near maximal efficacy based on JIA-ACR30 responses in other pJIA patient populations [44]. The mean $\mathrm{C}_{\text {trough }}$ was similar to that reported in a previous study of abatacept in Japanese patients with RA [45]. Since the efficacy and PK data presented here demonstrate a gradual cumulative benefit with continuous abatacept treatment, it is possible that continuing treatment -assuming that it is well tolerated - even if initially there is minimal or no benefit, may achieve a clinical response over time.

IV abatacept was well tolerated and no new safety signals were identified. The AE profile included events of special interest (infections, malignancies, autoimmune disorders, and infusion reactions) and was comparable with that reported previously for abatacept in patients with pJIA [12, 15, 26], indicating that the safety profile does not differ markedly between Japanese patients and other populations.

No ADAs were detected during the short-term period and only one patient had a positive immunogenic response during the cumulative period. This positive response did not appear to be associated with disease flare, SAEs, acute infusional AEs, hypersensitivity, autoimmune disorders, or low abatacept serum concentrations; importantly, JIA-ACR30, 50, and 70 responses were achieved in this patient. These results are consistent with previous reports of patients with pIIA testing positive for ADAs where no effects on efficacy, safety, or PK were observed [12].

Limitations to this study should be noted. First, the small sample size should be considered when interpreting the results. Furthermore, this was an open-label, single-arm study, without a placebo or other active treatment arm for comparison of the efficacy and safety of abatacept in this Japanese patient population. However, the findings presented here are consistent with those from other studies in JIA and with the large body of abatacept clinical data available for adult patients with RA. Further studies of randomized design and on large numbers of patients may be warranted to support the results observed in this trial.

\section{Conclusions}

In this study of Japanese children and adolescents with pJIA, IV abatacept treatment was effective, with benefits accruing over time, and no new safety concerns were identified. These data suggest that IV abatacept treatment provides an effective and well-tolerated treatment option for Japanese patients with active pJIA who do not tolerate existing first-line treatment.

\section{Abbreviations}

ACR: American College of Rheumatology; ACR: American College of Rheumatology; ADA: Anti-drug antibodies; AE: Adverse event; CHAQDI: Childhood Health Assessment Questionnaire-Disability Index; Cls: Confidence intervals; CRP: C-reactive protein; CTLA4: Cytotoxic Tlymphocyte-associated protein-4; DMARD: Disease-modifying anti-rheumatic drug; ICORE: Institute of Clinical Outcomes Research and Education; Ig: Immunoglobulin; IL: Interleukin; IV: Intravenous; JADAS27-CRP: Juvenile 
arthritis disease activity score 27 active joint count-CRP; JADAS27CRP: Juvenile Arthritis Disease Activity Score in 27 joints using C-reactive protein; JIA: Juvenile idiopathic arthritis; JIA-ACR30: JIA-American College of Rheumatology criteria 30; LOM: Limitation of motion; MDA: Minimal disease activity; MedDRA: Medical Dictionary for Regulatory Activities version; MTX: Methotrexate; PaGA: Parental Global Assessment; pJIA: Polyarticularcourse juvenile idiopathic arthritis; PK: Pharmacokinetics; RA: Rheumatoid arthritis; RF: Rheumatoid factor; SAEs: Serious AEs; TNFi: Tumor necrosis factor-a inhibitor

\section{Acknowledgements}

The study was sponsored by Bristol-Myers Squibb K.K. and Ono Pharmaceutical Co., Ltd. Professional medical writing and editorial assistance was provided by Natalie Griffiths, PhD, and Katerina Kumpan, PhD, at Caudex, and was funded by Bristol-Myers Squibb K.K. and Ono Pharmaceutical Co., Ltd.

\section{Funding}

This clinical study was sponsored by Bristol-Myers Squibb K.K. and Ono Pharmaceutical Co., Ltd.

\section{Availability of data and materials}

Bristol-Myers Squibb policy on data sharing may be found at https://www. bms.com/researchers-and-partners/clinical-trials-and-research/disclosurecommitment.htm

\section{Authors' contributions}

All authors were involved in drafting the article or revising it critically for important intellectual content, and all authors approved the final version to be published. Study conception and design: ST, NA, YT, MM, YI, SY. Acquisition of data: $\mathrm{RH}, \mathrm{HU}, \mathrm{ST}, \mathrm{NO}, \mathrm{NI}, \mathrm{YY}, \mathrm{YN}, \mathrm{TK}, \mathrm{IK}, \mathrm{TI}, \mathrm{NK}$. Analysis and interpretation of data: $\mathrm{RH}, \mathrm{ST}, \mathrm{NA}, \mathrm{YT}, \mathrm{MM}, \mathrm{YI}, \mathrm{SY}$

\section{Ethics approval and consent to participate}

This study was conducted in accordance with the Declaration of Helsinki, the International Conference on Harmonisation Guidelines for Good Clinical Practice, and local regulations. An institutional review board or independent ethics committee at each site approved the protocol, consent form, and any other written information provided to patients or their legal representatives.

\section{Consent for publication}

Not applicable

\section{Competing interests}

M.M. received research grants from Chugai Pharmaceutical Co., Ltd., Ono Pharmaceutical Co., Ltd. Mitsubishi Tanabe Pharma, UCB Japan Co., Ltd., CSL Behring, Towa Pharmaceutical Co., Ltd., AbbVie Japan Co., Ltd., Japan Blood Products Organization, Ayumi Pharmaceutical Co. and Nippon Kayaku Co. Ltd.; speaker fees from MSD K.K; and consulting fees from Daiichi Sankyo Co., Ltd. and Taisho Pharmaceutical Co., Ltd. N.A. and Y.T. are employees of BristolMyers Squibb K.K. All other authors declare no conflicts of interest associated with this manuscript.

\section{Publisher's Note}

Springer Nature remains neutral with regard to jurisdictional claims in published maps and institutional affiliations.

\footnotetext{
Author details

'Department of Pediatrics, Yokohama City University School of Medicine, 3-9 Fukuura, Kanazawa-ku, Yokohama-shi, Kanagawa 236-0004, Japan. 2Department of General Pediatrics, Miyagi Children's Hospital, 4-3-17 Ochiai, Aoba-ku, Sendai-shi, Miyagi 989-3126, Japan. ${ }^{3}$ Department of Pediatrics, Kagoshima University Medical and Dental Hospital, 8-35-1 Sakuragaoka, Kagoshima-shi, Kagoshima 890-8544, Japan. ${ }^{4}$ Department of Pediatrics, Graduate School of Medicine, Osaka Medical College, 2-7 Daigaku-machi, Takatsuki-shi, Osaka 569-8686, Japan. ${ }^{5}$ Department of Immunology and Infectious Diseases, Aichi Children's Health and Medical Center, 1-2 Osakata, Morioka-cho, Oobu-shi, Aichi 474-8710, Japan. ${ }^{6}$ Department of Pediatric Rheumatology, Hyogo Prefectural Kobe Children's Hospital, 1-6-7 Minamimachi, Minatojima, Chuo-ku, Kobe-shi, Hyogo 650-0047, Japan. DDepartment of Pediatrics, Japan Community Health Care Organization Sapporo Hokushin Hospital, 1-2, 2-jo, 6-chrome, Atsubetsu-chuo,
}

Atsubetsu-ku, Sapporo-shi, Hokkaido 004-8618, Japan. ${ }^{8}$ Department of Pediatrics, Hokkaido University Hospital, North-15, West-7, Sapporo-shi, Hokkaido 060-8638, Japan. ${ }^{9}$ Division of Infection, Immunology and Rheumatology, Kanagawa Prefectural Hospital Organisation Kanagawa Children's Medical Center, 2-138-4 Mutsukawa, Minami-ku, Yokohama-shi, Kanagawa 232-8555, Japan. ${ }^{10}$ Department of Pediatrics, University of the Ryukyus Hospital, 207 Aza Uehara, Nishihara-cho, Nakagami-gun, Okinawa 903-0215, Japan. ${ }^{11}$ Bristol-Myers Squibb K.K, 6-5-1 Nishi-Shinjuku, Shinjuku-ku, Tokyo 163-1328, Japan. ${ }^{12}$ Department of Lifetime Clinical Immunology, Graduate School of Medical and Dental Sciences, Tokyo Medical and Dental University, 1-5-45 Yushima, Bunkyo-ku, Tokyo 113-8510, Japan. ${ }^{13}$ Department of Pediatrics, Graduate School of Medicine, Nippon Medical School, 1-1-5 Sendagi, Bunkyo-ku, Tokyo 113-8603, Japan. ${ }^{14}$ Fuji Toranomon Orthopaedics Hospital, 1067-1 Kawashimata, Gotenba-shi, Shizuoka 412-0045, Japan.

Received: 17 October 2018 Accepted: 11 April 2019

Published online: 30 April 2019

\section{References}

1. Ravelli A, Martini A. Juvenile idiopathic arthritis. Lancet. 2007;369(9563):767-78.

2. Yokota S, Mori M, Imagawa T, Takei S, Murata T, Tomiita M, et al. Proposal for juvenile idiopathic arthritis guidance on diagnosis and treatment for primary care pediatricians and nonpediatric rheumatologists (2007). Mod Rheumatol. 2007:17(5):353-63.

3. Petty RE, Southwood TR, Manners P, Baum J, Glass DN, Goldenberg J, et al. International league of associations for rheumatology classification of juvenile idiopathic arthritis: second revision, Edmonton, 2001. J Rheumatol. 2004;31(2):390-2.

4. Rosenberg AM, Oen KG. Polyarticular juvenile idiopathic arthritis. In: Petty RE, Laxer RM, CB L, Wedderburn LR, editors. Textbook of pediatric rheumatology. 7th ed. Philadelphia: Elsevier Health Sciences; 2015. p. 217.

5. Beukelman T, Patkar NM, Saag KG, Tolleson-Rinehart S, Cron RQ, DeWitt EM, et al. 2011 American College of Rheumatology recommendations for the treatment of juvenile idiopathic arthritis: initiation and safety monitoring of therapeutic agents for the treatment of arthritis and systemic features. Arthritis Care Res (Hoboken). 2011;63(4):465-82.

6. Webb K, Wedderburn LR. Advances in the treatment of polyarticular juvenile idiopathic arthritis. Curr Opin Rheumatol. 2015;27(5):505-10.

7. Orencia Prescribing Information [in Japanese]2017 2/2/2018. Available from: https://www.pmda.go.jp/PmdaSearch/iyakuSearch/.

8. Humira Prescribing Information 2017 2/2/2018. Available from: http://www. pmda.go.jp/files/000224547.pdf.

9. Actemra Prescribing Information 2017 2/2/2018. Available from: http://www. pmda.go.jp/files/000224730.pdf.

10. Brunner HI, Ruperto N, Zuber Z, Keane C, Harari O, Kenwright A, et al. Efficacy and safety of tocilizumab in patients with polyarticular-course juvenile idiopathic arthritis: results from a phase 3, randomised, doubleblind withdrawal trial. Ann Rheum Dis. 2015:74:1110-7.

11. Brunner HI, Ruperto N, Tzaribachev N, Horneff G, Chasnyk VG, Panaviene V, et al. Subcutaneous golimumab for children with active polyarticular-course juvenile idiopathic arthritis: results of a multicentre, double-blind, randomised-withdrawal trial. Ann Rheum Dis. 2018;77(1):21-9.

12. Lovell D, Ruperto N, Mouy R, Paz E, Rubio-Pérez N, Silva CA, et al. Long-term safety, efficacy, and quality of life in patients with juvenile idiopathic arthritis treated with intravenous abatacept for up to seven years. Arthritis Rheumatol. 2015:67(10):2759-70.

13. Lovell DJ, Giannini EH, Reiff A, Cawkwell GD, Silverman ED, Nocton JJ, et al. Etanercept in children with polyarticular juvenile rheumatoid arthritis. Pediatric Rheumatology Collaborative Study Group. N Engl J Med. 2000; 342(11):763-9.

14. Lovell DJ, Ruperto N, Goodman S, Reiff A, Jung L, Jarosova K, et al. Adalimumab with or without methotrexate in juvenile rheumatoid arthritis. N Engl J Med. 2008;359(8):810-20.

15. Ruperto N, Lovell DJ, Quartier P, Paz E, Rubio-Perez N, Silva CA, et al. Longterm safety and efficacy of abatacept in children with juvenile idiopathic arthritis. Arthritis Rheum. 2010;62(6):1792-802.

16. Silverman E, Spiegel L, Hawkins D, Petty R, Goldsmith D, Schanberg L, et al. Long-term open-label preliminary study of the safety and efficacy of leflunomide in patients with polyarticular-course juvenile rheumatoid arthritis. Arthritis Rheum. 2005;52(2):554-62. 
17. Silverman E, Mouy R, Spiegel L, Jung LK, Saurenmann RK, Lahdenne P, et al. Leflunomide or methotrexate for juvenile rheumatoid arthritis. N Engl J Med. 2005;352(16):1655-66.

18. Vander Cruyssen B, Van Looy S, Wyns B, Westhovens R, Durez P, Van den Bosch $F$, et al. Four-year follow-up of infliximab therapy in rheumatoid arthritis patients with long-standing refractory disease: attrition and longterm evolution of disease activity. Arthritis Res Ther. 2006;8(4):R112.

19. Moreland LW, Alten R, Van den Bosch F, Appelboom T, Leon M, Emery P, et al. Costimulatory blockade in patients with rheumatoid arthritis: a pilot, dose-finding, double-blind, placebo-controlled clinical trial evaluating CTLA$4 \mathrm{lg}$ and LEA29Y eighty-five days after the first infusion. Arthritis Rheum. 2002;46(6):1470-9.

20. Orencia Prescribing Information2017 February 2, 2018. Available from: http://packageinserts.bms.com/pi/pi_orencia.pdf.

21. Cutolo M, Sulli A, Paolino S, Pizzorni C. CTLA-4 blockade in the treatment of rheumatoid arthritis: an update. Expert Rev Clin Immunol. 2016;12(4):417-25.

22. Genovese MC, Schiff M, Luggen M, Becker JC, Aranda R, Teng J, et al. Efficacy and safety of the selective co-stimulation modulator abatacept following 2 years of treatment in patients with rheumatoid arthritis and an inadequate response to anti-tumour necrosis factor therapy. Ann Rheum Dis. 2008;67(4):547-54

23. Kremer JM, Genant HK, Moreland LW, Russell AS, Emery P, Abud-Mendoza C, et al. Results of a two-year follow up study of patients with rheumatoid arthritis who received a combination of abatacept and methotrexate. Arthritis Rheum. 2008;58(4):953-63.

24. Westhovens R, Kremer JM, Moreland LW, Emery P, Russell AS, Li T, et al. Safety and efficacy of the selective costimulation modulator abatacept in patients with rheumatoid arthritis receiving background methotrexate: a 5year extended phase IIB study. J Rheumatol. 2009;36(4):736-42.

25. Lovell D, Ruperto N, Tzaribachev N, Zeft A, Cimaz R, Stanevica V, et al. Long term effectiveness and safety of abatacept in juvenile idiopathic arthritis: interim results from the abatacept in JIA registry [abstract]. Arthritis Rheumatol. 2017;69(Suppl 10):Abstract 2272.

26. Ruperto N, Lovell DJ, Quartier P, Paz E, Rubio-Perez N, Silva CA, et al. Abatacept in children with juvenile idiopathic arthritis: a randomised, double-blind, placebo-controlled withdrawal trial. Lancet. 2008;372(9636): 383-91.

27. Bristol-Myers Squibb Canada. Orencia (abatacept) product monograph 2017 [Available from: http://www.bms.com/assets/bms/ca/documents/ productmonograph/ORENCIA_EN_PM.pdf.

28. Orencia $250 \mathrm{mg}$ powder for concentrate for solution for infusion 2017 [Available from: http://www.medicines.org.uk/emc/medicine/19714/SPC/.

29. Bristol-Myers Squibb K.K., Ono Pharmaceutical Co. L. Orencia ${ }^{\oplus}$ for Intravenous Infusion 250 mg, Selective T-cell Co-stimulation Modulator: Approval for Additional Indication of Active Polyarticular Juvenile Idiopathic Arthritis for Partial Change in Approved items of Manufacturing and Marketing Approval in Japan 2018 [Available from: https://www.ono.co.jp/ eng/news/pdf/sm_cn180223.pdf.

30. Giannini EH, Ruperto N, Ravelli A, Lovell DJ, Felson DT, Martini A. Preliminary definition of improvement in juvenile arthritis. Arthritis Rheum. 1997;40(7): 1202-9.

31. Wallace CA, Giannini EH, B H, L I, Ruperto N. American College of Rheumatology provisional criteria for defining clinical inactive disease in select categories of juvenile idiopathic arthritis. Arthritis Care Res (Hoboken). 2011;63(7):929-36.

32. Ruperto N, Ravelli A, Pistorio A, Malattia C, Cavuto S, Gado-West L, et al. Cross-cultural adaptation and psychometric evaluation of the childhood health assessment questionnaire (CHAQ) and the child health questionnaire $(\mathrm{CHQ})$ in 32 countries. Review of the general methodology. Clin Exp Rheumatol. 2001;19(4 Suppl 23):S1-9.

33. Consolaro A, Ruperto N, Bazso A, Pistorio A, Magni-Manzoni S, Filocamo G, et al. Development and validation of a composite disease activity score for juvenile idiopathic arthritis. Arthritis Rheum. 2009;61(5):658-66.

34. Consolaro A, Bracciolini G, Ruperto N, Pistorio A, Magni-Manzoni S, Malattia $C$, et al. Remission, minimal disease activity, and acceptable symptom state in juvenile idiopathic arthritis: defining criteria based on the juvenile arthritis disease activity score. Arthritis Rheum. 2012;64(7):2366-74.

35. Mourao AF, Santos MJ, Melo-Gomes J, Martins FM, Costa JA, Ramos F, et al. Using the juvenile arthritis disease activity score based on erythrocyte sedimentation rate or C-reactive protein level: results from the Portuguese register. Arthritis Care Res (Hoboken). 2014;66(4):585-91.
36. Nordal EB, Zak M, Aalto K, Berntson L, Fasth A, Herlin T, et al. Validity and predictive ability of the juvenile arthritis disease activity score based on CRP versus ESR in a Nordic population-based setting. Ann Rheum Dis. 2012; 71(7):1122-7.

37. Ruperto N, Pistorio A, Ravelli A, Hasija R, Guseinova D, Filocamo G, et al. Criteria to define response to therapy in paediatric rheumatic diseases. Eur J Clin Pharmacol. 2011;67(Suppl 1):125-31.

38. European Medicines Agency. Assessment Report: Orenica; Procedure No. EMEA/H/C/000701/II/01052017 2/2/2018. Available from: http://www.ema. europa.eu/docs/en_GB/document_library/EPAR_-_Assessment_Report_-_ Variation/human/000701/WC500233148.pdf.

39. Haggerty HG, Abbott MA, Reilly TP, DeVona DA, Gleason CR, Tay L, et al. Evaluation of immunogenicity of the $T$ cell costimulation modulator abatacept in patients treated for rheumatoid arthritis. J Rheumatol. 2007; 34(12):2365-73.

40. Imagawa T, Yokota S, Mori M, Miyamae T, Takei S, Imanaka H, et al. Safety and efficacy of tocilizumab, an anti-IL-6-receptor monoclonal antibody, in patients with polyarticular-course juvenile idiopathic arthritis. Mod Rheumatol. 2012;22(1):109-15.

41. Kuemmerle-Deschner JB, Benseler S. Abatacept in difficult-to-treat juvenile idiopathic arthritis. Biologics. 2008;2(4):865-74.

42. Sawyer MG, Carbone JA, Whitham JN, Roberton DM, Taplin JE, Varni JW et al. The relationship between health-related quality of life, pain, and coping strategies in juvenile arthritis--a one year prospective study. Qual Life Res. 2005;14(6):1585-98.

43. Consolaro A, Schiappapietra B, Dalpra' S, Calandra S, Martini A, Ravelli A Optimisation of disease assessments in juvenile idiopathic arthritis. Clinical Exp Rheumatol. 2014;32(Suppl. 85):S126-S30.

44. Li X, Passarell JA, Lin K, Roy A, Murthy B, Girgis IG. Population pharmacokinetics and exposure-response analyses for abatacept in juvenile idiopathic arthritis. American Conference on Pharmacometrics (ACoP8), Fort Lauderdale, FL, USA, 15-18 October, abstract W-069; 20172017.

45. Iwahashi M, Inoue $H$, Matsubara T, Tanaka T, Amano K, Kanamono T, et al. Efficacy, safety, pharmacokinetics, and immunogenicity of abatacept administered subcutaneously or intravenously in Japanese patients with rheumatoid arthritis and inadequate response to methotrexate: a phase II/II, randomized study. Mod Rheumatol. 2014;24(6):885-91.

\section{Ready to submit your research? Choose BMC and benefit from:}

- fast, convenient online submission

- thorough peer review by experienced researchers in your field

- rapid publication on acceptance

- support for research data, including large and complex data types

- gold Open Access which fosters wider collaboration and increased citations

- maximum visibility for your research: over $100 \mathrm{M}$ website views per year

At $\mathrm{BMC}$, research is always in progress.

Learn more biomedcentral.com/submissions 